\title{
Models for the Comet Dynamical Environment
}

\author{
Sharyl M. Byram,늘 Daniel J. Scheeres, $\_$and Michael R. Combi \\ University of Michigan, Ann Arbor, Michigan 48109 \\ DOI: $10.2514 / 1.28299$
}

\begin{abstract}
An outgassing jet model is presented in support of spacecraft navigation for future missions to comets. The outgassing jet is modeled as an emission cone while the comet is modeled as a uniform density triaxial ellipsoid. The comet's motion about the sun is included in the model. The model is used to explore the effects on a spacecraft passing through an outgassing jet field. The outgassing jet model is also used for simulation and estimation of the physical outgassing properties of jets at and near the surface of a comet. Methods for estimating the locations and sizes of multiple outgassing jets are presented.
\end{abstract}

\begin{tabular}{|c|c|}
\hline \multicolumn{2}{|r|}{ Nomenclature } \\
\hline$a$ & $=$ semimajor axis \\
\hline$a_{p}$ & $=$ outgassing jet acceleration vector \\
\hline$a_{t}, b_{t}, c_{t}$ & $=$ chord lengths in inscribed triangle \\
\hline$B$ & $=$ spacecraft mass-to-area ratio \\
\hline$c_{1}, c_{2}, c_{3}$ & $=$ constants \\
\hline$E$ & $=$ orbital energy \\
\hline$e$ & $=$ eccentricity \\
\hline$\hat{e}_{\text {jet }}$ & $=$ unit vector in direction of jet orientation \\
\hline$\hat{e}_{\text {surf }}$ & $\begin{array}{l}=\text { unit vector in direction of jet center on comet } \\
\text { surface }\end{array}$ \\
\hline$\hat{F}_{N}, \hat{F}_{R}, \hat{F}_{T}$ & $\begin{array}{l}=\text { impulses in normal, radial, and transverse } \\
\text { direction }\end{array}$ \\
\hline$f$ & $=$ thermal inertia function \\
\hline$g_{0}$ & $=$ constant \\
\hline $\boldsymbol{H}$ & $=$ angular momentum vector \\
\hline$i$ & $=$ inclination \\
\hline$I$ & $=$ inertia dyad \\
\hline$I_{d}$ & $=$ dynamic inertia \\
\hline$I_{x}, I_{y}, I_{z}$ & $=$ principle axes moments of inertia \\
\hline$J$ & $=$ jet cone half-angle cost function \\
\hline$K(k)$ & $=$ complete elliptic integral of the first kind \\
\hline$k, n$ & $=$ elliptic function parameters \\
\hline $\bar{M}$ & $=$ mean anomaly \\
\hline$P$ & $=$ comet period \\
\hline$p$ & $=$ orbital parameter \\
\hline$p$ & $=$ pressure vector \\
\hline$p_{0}$ & $=$ effective jet pressure at comet surface \\
\hline$Q_{j}$ & $=$ mass ejection rate per unit area \\
\hline$Q_{*}$ & $\begin{array}{l}=\text { mass ejection rate of plane with equal area to the } \\
\text { comet perpendicular to the sun at } 1 \mathrm{AU}\end{array}$ \\
\hline$q$ & $=$ radius of periapsis \\
\hline$R(t)$ & $=$ rotation matrix \\
\hline $\boldsymbol{r}$ & $=$ spacecraft position \\
\hline $\boldsymbol{r}_{j}$ & $=$ spacecraft position relative to jet's virtual center \\
\hline$r_{\text {og }}$ & $=$ jet outgassing centerline \\
\hline$r_{p}$ & $=$ jet surface cross-section's radius \\
\hline$r_{s}$ & $=$ comet heliocentric distance \\
\hline
\end{tabular}

Presented as Paper 6288 at the AIAA/AAS Astrodynamics Specialist Conference, Keystone, CO, 21-24 August 2006; received 12 October 2006; revision received 19 April 2007; accepted for publication 24 April 2007. Copyright $@ 2007$ by the American Institute of Aeronautics and Astronautics, Inc. All rights reserved. Copies of this paper may be made for personal or internal use, on condition that the copier pay the $\$ 10.00$ per-copy fee to the Copyright Clearance Center, Inc., 222 Rosewood Drive, Danvers, MA 01923; include the code 0731-5090/07 $\$ 10.00$ in correspondence with the CCC.

*Ph.D. Candidate, Department of Aerospace Engineering; byramsm@ umich.edu. Student Member AIAA.

${ }^{\dagger}$ Associate Professor, Department of Aerospace Engineering; scheeres@ umich.edu. Professional Member AIAA.

Research Professor, Department of Atmospheric, Oceanic, and Space Sciences;mcombi@umich.edu.

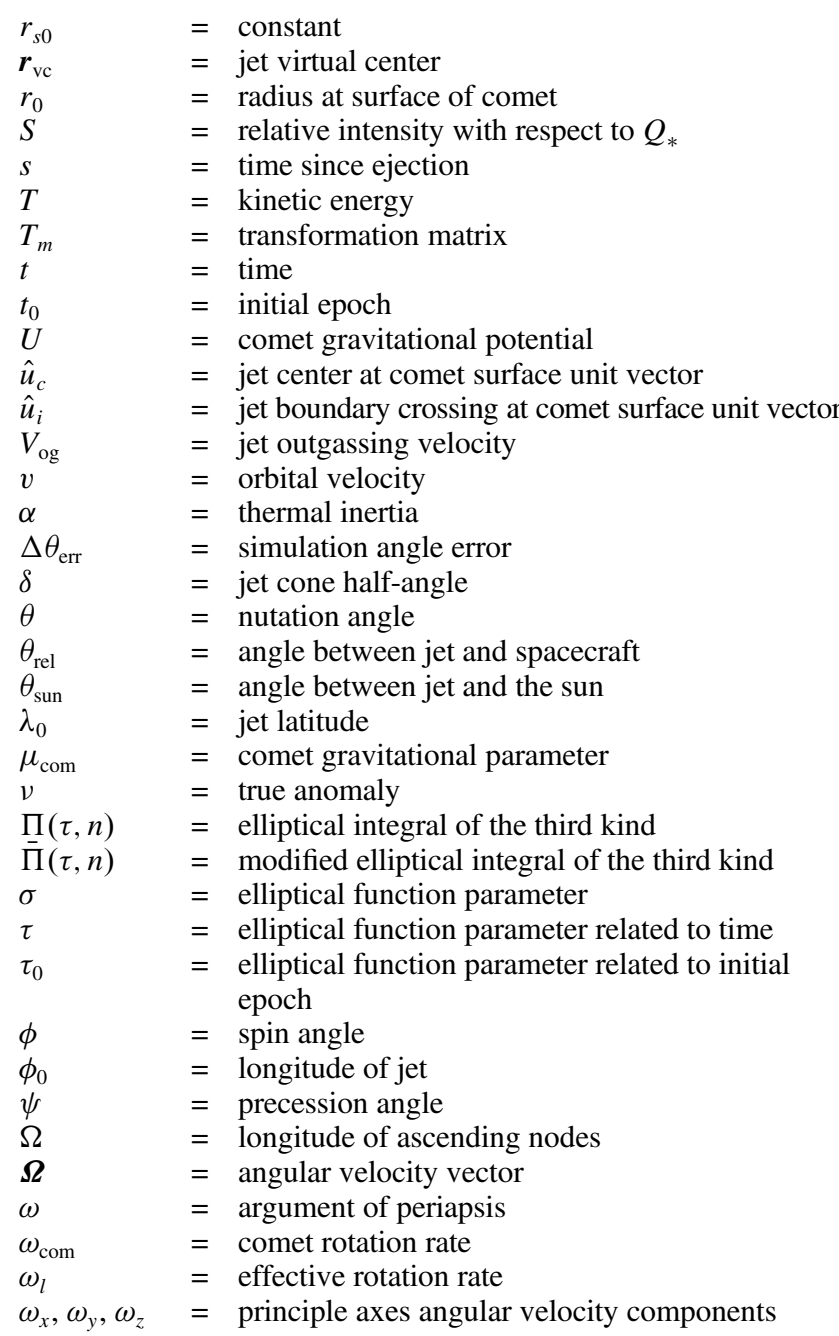

Prefix

$\Delta \quad=$ small change

\section{Introduction}

W ITH the return of the Stardust capsule back to Earth in January, the interest in cometary science has grown. The space agencies around the world have sent an increasing number of missions to comets in the solar system as well as to asteroids. NASA launched Stardust in 1999 which reached the comet P/Wild 2 in January 2004 and returned a sample of dust and volatiles from the coma of the comet. In July 2005, NASA's Deep Impact mission 
provided to the world high-resolution images of the surface of the comet Tempel 1 as well as impacting the surface to gain knowledge of its physical properties. ESA's Rosetta mission is scheduled to reach comet $67 \mathrm{P} /$ Churyumov-Gerasimenko in 2014 and deliver a lander to the surface. All of these missions can provide an abundance of scientific information about the physical properties of comets, but they are also of interest in the field of orbital dynamics.

Because of the similarity in shape and size, comets yield a set of dynamical problems common to that of asteroids, but it is the outgassing fields that make the dynamical environment of comets unique. Orbit determination of a spacecraft about an outgassing comet has been explored previously. Miller et al. [1] modeled the outgassing as constant bias nongravitational acceleration with a variable modeling error acceleration from an active region which is defined by a portion of the surface area of an ellipsoid. Weeks [2] modeled the outgassing as a combination of discrete jets and continuous surface sublimation with the acceleration due to the jets decreasing as the orbiting spacecraft's angle with the jet increased. Scheeres et al. [3] approached the outgassing acceleration in two ways to determine the stability of the spacecraft in terms of impacting or escaping from the comet. Their first outgassing model assumes that the outgassing field is continuous but variable depending on the angle made with the sun. Their second model assumes that the comet has jets emanating from the surface, with spacecraft interaction only acting in the vicinity of the jets.

It is the description of the outgassing jets and their dynamical implications that is the focus of this paper. There exist many theories about the structure of a comet's outgassing fields. Crifo et al. [4] theorize that the coma structure is produced by multiple interacting dusty gas jets. Sekanina et al. [5] use a model of comet 81P/Wild 2 from images taken by Stardust of dust particles, which are ejected from a small active source, which make up a thin conical sheet in the coma before the formation is gradually dispersed by various forces. They discuss 20 jets imaged on comet 81P/Wild 2 oriented in an arbitrary direction away from the surface. For the purpose of this research, it will be assumed that the outgassing pressure field is created by a collection of fixed active patch jets on the surface of the comet similar to the ones described in Sekanina et al. [5]. This model, unlike others, will have a full three-dimensional geometry. Also addressed is the need for a spacecraft to navigate these jet outgassing fields while in orbit about a comet by defining and exploring a simple model for an outgassing jet while considering its implications. In addition to the model and simulation, it is discussed how the parameters of this model can be estimated using navigational data from a spacecraft's passages through the jet's outgassing field. In particular, this model uses the jets identified by Sekanina et al. [5] to simulate a spacecraft's trajectory about an idealized comet $\overline{8} 1 / \mathrm{P}$ Wild2.

The model and navigation techniques developed in this paper are key for predicting jet locations and in supporting navigation strategies for spacecraft missions to comets. Currently, ESA's Rosetta mission is in transit to comet $67 \mathrm{P} /$ Churyumov-Gerasimenko and would be a prime application of this model. It is also a crucial navigation technology for the design of proposed comet sample return missions. In the latest NASA solar system exploration road map [6], comet surface sample return is identified as one of the key New Frontiers missions for launch in the next decade. In particular, the sample is to come from an active patch on the surface. This research directly addresses crucial navigation capabilities for the development and implementation of such missions.

\section{Equations of Motion}

To begin, it is assumed that the comet is located significantly far from any other major celestial body such that the spacecraft's motion about the comet follows the two-body orbital equations. The mass of the spacecraft is assumed to be significantly smaller than that of the comet, such that the equations of motion are of the form:

$$
\ddot{\boldsymbol{r}}=\frac{\partial U}{\partial \boldsymbol{r}}+\boldsymbol{a}_{p}
$$

where $\boldsymbol{r}$ is the spacecraft's position vector relative to the comet's center of mass, $U$ is the comet's gravitational potential, and $\boldsymbol{a}_{p}$ is the outgassing jet acceleration applied to the spacecraft when the spacecraft is determined to be within a jet's outgassing field. It is assumed that the jet is oriented in some arbitrary direction relative to the comet fixed frame and will diminish as $1 /\left|\boldsymbol{r}_{j}\right|^{2}$, where $\boldsymbol{r}_{j}=\boldsymbol{r}-$ $\boldsymbol{r}_{\mathrm{vc}}$ is the spacecraft's position vector relative to the virtual center of the jet, $\boldsymbol{r}_{\mathrm{vc}}$. This assumption leads to the basic equation for the outgassing acceleration:

$$
\boldsymbol{a}_{p}=\frac{p_{0}\left(\boldsymbol{r}_{j}\right)}{B} \frac{\boldsymbol{r}_{j}}{\left|\boldsymbol{r}_{j}\right|^{3}}
$$

where $B$ is the spacecraft's mass-to-area ratio and $p_{0}\left(\boldsymbol{r}_{j}\right)$ is the effective pressure field at the comet surface. Equation (2) provides the basis of the jet dynamical outgassing model.

\section{Outgassing Jet Model}

This section defines and explores the geometry of an emission cone model for an outgassing jet from the surface of a comet. Given is a detailed description based on Stardust's data of the Wild 2 comet [5], as well as a general description of this model and its implications. First presented is the basic definition and features of the model.

\section{A. Comet Assumptions}

The comet is assumed be an ellipsoid with principle half-lengths which represent the best fit to images taken in respect to size and shape. The mass distribution of this comet model is assumed to have uniform mass density. This, however, is only an approximation to the true comet body. Scheeres [7] describes the gravitational potential for an ellipsoid used in the equations of motion. The rotational properties of the comet body are assumed to be constant rotation, $\omega_{\text {com }}$, about the body-fixed $z$ axis which may be inclined to the comet's orbital plane. For an ellipsoid with equal principle halflengths, the model collapses to the special case of a spherical shape which yields a simple point mass gravity field. This model can easily be generalized to the case of nonprincipal axis rotation; however, this case is not presented here.

\section{B. Outgassing Jet}

In the mathematical model, the outgassing field of the comet is assumed to be produced by a single jet located on the surface of the comet, although the simulated model includes multiple jets of varying strength. The jet is fixed on the comet surface with its center located at a radius of $r_{0}$, longitude of $\phi_{0}$, and latitude of $\lambda_{0}$ in the body-fixed frame.

The active region on the surface of the comet is assumed to have a circular cross section where the size and shape of the jet is defined by a constant half-angle $\delta$, which represents the maximum extent of the outgassing region and surface radius $r_{p}$. These two parameters along with the jet orientation define the location of the virtual center of the jet $\boldsymbol{r}_{\mathrm{vc}}$, seen in Fig. 1. The jet is assumed to continuously eject gas while illuminated by the sun at a constant velocity $V_{\text {og }}$, greater than the escape velocity for the comet in a direction defined by the jet's orientation, which may point in any arbitrary direction away from the comet surface. Note that the assumption of constant velocity may not hold close to the comet surface where complex gas dynamics and interactions are occurring [8]. The centerline of an outgassing jet becomes a continuous function of time since ejection $s$ and can be expressed in the comet body-fixed frame (illustrated in Fig. 2):

$$
\boldsymbol{r}_{\mathrm{og}}(s)=r_{0} \hat{e}_{r_{\text {surf }}}+V_{\mathrm{og}} s \hat{e}_{r_{\mathrm{jet}}}
$$

where $\hat{e}_{r_{\text {surf }}}$ is the body-fixed unit vector pointing from the center of the comet to the jet center point on the surface and $\hat{e}_{r_{\mathrm{jet}}}$ is the bodyfixed unit vector pointing in the jet's orientation direction. This frame rotates with the comet at a constant rate $\omega_{\text {com }}$ and is transformed from a nonrotating coordinate system with $z$ axis aligned along the spin axis of the comet, to the body-fixed rotating coordinate frame by 


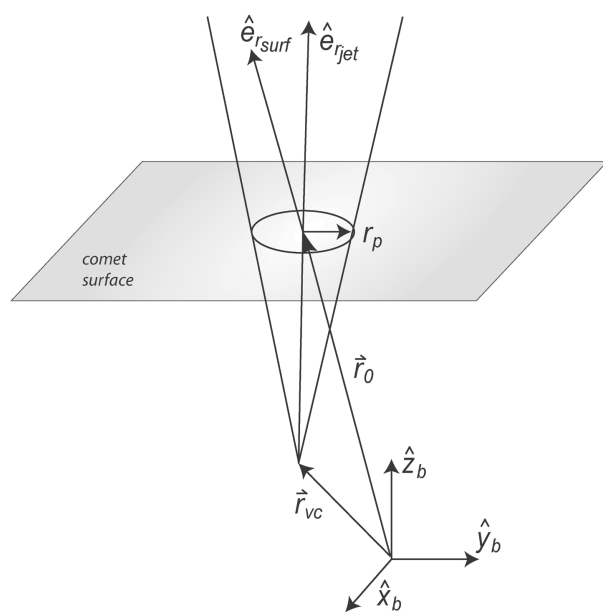

Fig. 1 Outgassing jet surface.

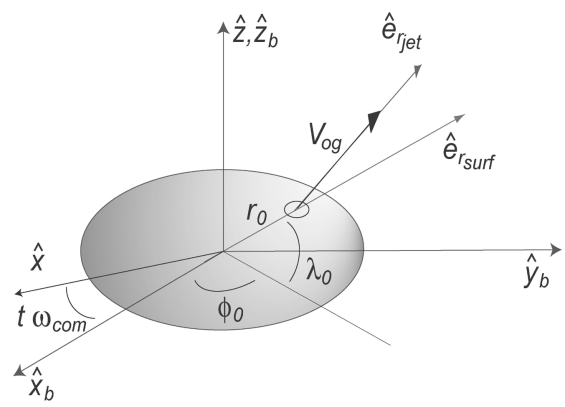

Fig. 2 Coordinate frames.

$R(t)$

$$
=\left[\begin{array}{ccc}
\cos \left(\lambda_{0}\right) \cos \left(\phi_{0}+\omega_{\text {com }} t\right) & \cos \left(\lambda_{0}\right) \sin \left(\phi_{0}+\omega_{\text {com }} t\right) & \sin \left(\lambda_{0}\right) \\
-\sin \left(\phi_{0}+\omega_{\text {com }} t\right) & \cos \left(\phi_{0}+\omega_{\text {com }} t\right) & 0 \\
-\sin \left(\lambda_{0}\right) \cos \left(\phi_{0}+\omega_{\text {com }} t\right) & -\sin \left(\lambda_{0}\right) \sin \left(\phi_{0}+\omega_{\text {com }} t\right) & \cos \left(\lambda_{0}\right)
\end{array}\right]
$$

The jet outgassing centerline is then expressed as a function of both the time $t$ as well as the time since ejection $s$.

$$
\boldsymbol{r}_{\mathrm{og}}(t, s)=R(t)^{T}\left[r_{0} \hat{e}_{r_{\text {surf }}}+V_{\mathrm{og}} s \hat{e}_{r_{\mathrm{jet}}}\right]
$$

Because a circular cross section is assumed, the surface of the outgassing jet is modeled as a solid cone that is defined by the constant half-angle $\delta$ from the centerline as well as the time since ejection $s$ in the body-fixed frame. This outgassing jet will generate a pressure field which is a function of the mass ejection rate per unit area of the jet at the surface of the comet $Q_{j}$ and the velocity of the material being ejected $V_{\mathrm{og}}$. Therefore the pressure of the outgassing at the surface of the comet becomes

$$
p_{0}=Q_{j} V_{\mathrm{og}}
$$

As the velocity field is assumed to be uniformly outward, the pressure vector is aligned with the velocity field in the direction of the jet's orientation as

$$
\boldsymbol{p}_{0}=Q_{j} V_{\mathrm{og}} \hat{e}_{r_{\mathrm{jet}}}
$$

The mass ejection rate of the jet, $Q_{j}$ can be estimated as [9]

$$
Q_{j}=S f\left(\theta_{\text {sun }}\right) g\left(r_{s}\right) Q_{*}
$$

where $Q_{*}$ is the mass ejection rate of an area equal to the surface area of the comet perpendicular to the sun at a distance of $1 \mathrm{AU}$ (astronomical unit) away, $S$ is the relative intensity with respect to $Q_{*}, \theta_{\text {sun }}$ is the angle between the body-fixed vector pointing in the direction of the sun and the body-fixed vector in the direction of the jet's center at the surface of the comet, and $r_{s}$ is the heliocentric distance of the comet. As the comet travels closer to the sun its activity will increase as a function of $r_{s}$. This outgassing strength has been empirically determined to follow the law:

$$
g\left(r_{s}\right)=g_{0}\left(\frac{r_{s}}{r_{s 0}}\right)^{-c_{1}}\left[1+\left(\frac{r_{s}}{r_{s 0}}\right)^{c_{2}}\right]^{-c_{3}}
$$

where $c_{1}=2.15, c_{2}=5.093, c_{3}=4.6142, r_{s 0}=2.808$, and $g_{0}=$ 0.111262 [10]. The strength of the pressure at the surface of the comet is related to the angle the sun makes with the jet, given by $f\left(\theta_{\text {sun }}\right)$. If the body-fixed vector pointing to the sun is defined as $\hat{u}_{s}$, then $\cos \theta_{\text {sun }}=\hat{u}_{s} \cdot \hat{r}_{\text {og }}(t, s)$. To account for a strong pressure when the surface is illuminated by the sun and a weak (possibly zero) pressure when it is not, this function takes the form:

$$
f\left(\theta_{\text {sun }}\right)=\max \left\{\begin{array}{l}
0 \\
1-\alpha\left(1-\cos \theta_{\text {sun }}\right)
\end{array}\right.
$$

where the parameter $\alpha$ is related to the thermal inertia and can have any value such that $0 \leq \alpha \leq 1$. The pressure magnitude felt by the orbiter is assumed to be inversely proportional to the relative distance from the virtual center of the jet, $\boldsymbol{r}_{j}=\boldsymbol{r}-\boldsymbol{r}_{\mathrm{vc}}$, such that the pressure vector at the orbiter's position becomes

$$
\boldsymbol{p}=\boldsymbol{p}_{0}\left(\frac{r_{0}}{\left|\boldsymbol{r}_{j}\right|}\right)^{2}
$$

where $\left|\boldsymbol{r}_{j}\right|>r_{0}$ is assumed. Then the acceleration felt by the spacecraft from the outgassing pressure used in Eq. (1) is of the form:

$$
\boldsymbol{a}_{p}=\frac{\boldsymbol{p}}{B}=\boldsymbol{p}_{0} \frac{1}{B}\left(\frac{r_{0}}{\left|\boldsymbol{r}_{j}\right|}\right)^{2}
$$

where $B$ is the ratio of the mass of the spacecraft to the area of the spacecraft normal to the comet.

\section{Orbit Mechanics About an Outgassing Jet}

\section{A. Orbital Elements}

To predict the trajectory of a spacecraft that passes through a jet outgassing field, start with the analysis of small changes in orbital elements. First, note that for large distances from the comet, the radial component of the outgassing is dominant and therefore the outgassing due to a jet passage will be considered to be a radial impulse. To begin, consider the orbital parameter $p$ and the orbital energy $E$ :

$$
\begin{gathered}
p=a\left(1-e^{2}\right) \\
E=-\frac{\mu_{\mathrm{com}}}{2 a}
\end{gathered}
$$

First, note that $p$ will be conserved [3] through a jet passage as it is related to the angular momentum which does not change under a radial impulse. Taking a small change in $p, \Delta p$, and in $E, \Delta E$, yields the following:

$$
\begin{gathered}
\Delta p=\Delta a\left(1-e^{2}\right)-2 a e \Delta e=0 \\
\Delta E=\frac{\mu_{\mathrm{com}} \Delta a}{2 a^{2}}
\end{gathered}
$$

The change in the orbital energy is used to find a small change in semimajor axis $a$.

$$
\Delta a=\frac{2 a^{2} \Delta E}{\mu_{\mathrm{com}}}
$$

It is seen in Eq. (17) that a positive change in energy increases the semimajor axis and that a negative change in energy results in a 
decrease in the semimajor axis. Substituting this result into Eq. (15) yields that a positive change in energy increases the eccentricity and that a negative change in energy results in a decrease in the eccentricity.

$$
\begin{gathered}
0=\frac{2 a^{2} \Delta E}{\mu_{\mathrm{com}}}\left(1-e^{2}\right)-2 a e \Delta e \\
\Delta e=\frac{a \Delta E}{\mu_{\mathrm{com}} e}\left(1-e^{2}\right)
\end{gathered}
$$

In terms of a jet passage, the energy equation as a function of velocity provides additional insight.

$$
E=\frac{1}{2} v^{2}-\frac{\mu_{\mathrm{com}}}{|\boldsymbol{r}|}
$$

where $v$ is the spacecraft's velocity magnitude. Variations in energy will arise due to impulsive changes in the spacecraft's velocity:

$$
\Delta E=v \Delta v
$$

Substituting this $\Delta E$ result in Eqs. (17) and (19) yields

$$
\begin{gathered}
\Delta a=\frac{2 a^{2} v \Delta v}{\mu_{\mathrm{com}}} \\
\Delta e=\frac{a v \Delta v}{\mu_{\mathrm{com}} e}\left(1-e^{2}\right)
\end{gathered}
$$

From Eqs. (22) and (23) it can be seen that a positive change in velocity increases the semimajor axis and eccentricity and that a negative change in velocity results in a decrease in the semimajor axis and eccentricity. Because the outgassing is assumed to primarily act in the radial direction, the radial component of the spacecraft's velocity is the only component affected. Therefore, if the spacecraft has a negative radial velocity component (Fig. 3) such as when it is traveling from apoapsis to periapsis, the outgassing jet will tend to circularize the orbit, or decrease the semimajor axis and eccentricity. Likewise, if the spacecraft has a positive radial velocity component such as when it is traveling from periapsis to apoapsis, the outgassing jet will tend to make the orbit more eccentric and increase the semimajor axis.

The radius of periapsis $q$ is also of interest. Taking a small change in the radius of the periapsis yields

$$
\begin{gathered}
q=a(1-e) \\
\Delta q=\Delta a(1-e)-a \Delta e
\end{gathered}
$$

This can be related to a change in velocity by substituting in Eqs. (22) and (23).

$$
\Delta q=\frac{-q^{2} v \Delta v}{\mu_{\mathrm{com}} e}
$$

This results in an increase in the radius of the periapsis when the spacecraft has a negative radial velocity component during a jet passage. Likewise, a decrease in the radius of the periapsis occurs

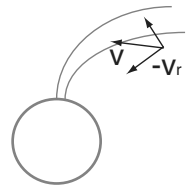

a)

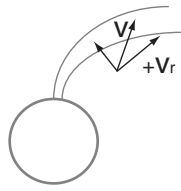

b)
Fig. 3 Spacecraft velocity components: a) negative radial component; b) positive radial component. when the spacecraft has a positive radial velocity component during a jet passage.

\section{B. Lagrange Planetary Equations}

Changes in the full set of orbital elements can be explored by considering the Lagrange planetary equations. Starting with these equations in the Gaussian form:

$$
\begin{gathered}
\frac{\mathrm{d} a}{\mathrm{~d} t}=\frac{2 a^{2} e}{h} \sin \nu \hat{F}_{R}+\frac{2 a^{2} h}{\mu_{\mathrm{com}} r} \hat{F}_{T} \\
\frac{\mathrm{d} e}{\mathrm{~d} t}=\frac{h}{\mu_{\mathrm{com}}}\left(\sin \nu \hat{F}_{R}+\frac{e+2 \cos \nu+e \cos ^{2} \nu}{1+e \cos / n u} \hat{F}_{T}\right) \\
\frac{\mathrm{d} i}{\mathrm{~d} t}=\frac{r}{h} \cos \varpi \hat{F}_{N} \\
\frac{\mathrm{d} \Omega}{\mathrm{d} t}=\frac{r \sin \varpi}{h \sin i} \hat{F}_{N}
\end{gathered}
$$

$\frac{\mathrm{d} \omega}{\mathrm{d} t}=-\frac{h}{\mu_{\mathrm{com}} e} \cos \nu \hat{F}_{R}-\frac{r}{h} \cot i \sin \varpi \hat{F}_{N}+\frac{\left(h^{2}+r \mu_{\mathrm{com}}\right) \sin v}{\mu_{\mathrm{com}} e h} \hat{F}_{T}$

$$
\begin{gathered}
\frac{\mathrm{d} \bar{M}}{\mathrm{~d} t}=n-\frac{1}{n a}\left(\frac{2 r}{a}-\frac{\left(1-e^{2}\right)}{e} \cos \nu\right) \hat{F}_{R} \\
-\frac{\left(1-e^{2}\right)}{n a e}\left(1+\frac{r}{p}\right) \sin \nu \hat{F}_{T}
\end{gathered}
$$

where

$$
\begin{gathered}
h=n a^{2} \sqrt{1-e^{2}} \\
n=\sqrt{\frac{\mu_{\mathrm{com}}}{a^{3}}} \\
\varpi=\omega+v \\
r=|\boldsymbol{r}|
\end{gathered}
$$

and $\hat{F}_{R}, \hat{F}_{T}$, and $\hat{F}_{N}$ are accelerations in the radial, transverse, and orbit normal directions, respectively, and $v$ is the true anomaly. For jet passages, it is assumed that the outgassing component in the radial direction is dominant; therefore the acceleration terms in Eqs. (2732) become

$$
\begin{gathered}
\hat{F}_{R}=a_{p}=p_{0} \frac{1}{B}\left(\frac{r_{0}}{\left|\boldsymbol{r}_{j}\right|}\right)^{2} \\
\hat{F}_{T}=0 \\
\hat{F}_{N}=0
\end{gathered}
$$

where $r_{0}$ is the comet radius at the jet location, and $\boldsymbol{r}_{j}$ is the position of the spacecraft relative to the virtual center of the jet. The Lagrange planetary equations for the outgassing acceleration become

$$
\frac{\mathrm{d} a}{\mathrm{~d} t}=\frac{2 a^{2} e}{h} \sin v p_{0} \frac{1}{B}\left(\frac{r_{0}}{\left|\boldsymbol{r}_{j}\right|}\right)^{2}
$$




$$
\begin{gathered}
\frac{\mathrm{d} e}{\mathrm{~d} t}=\frac{h}{\mu_{\mathrm{com}}} \sin v p_{0} \frac{1}{B}\left(\frac{r_{0}}{\left|\boldsymbol{r}_{j}\right|}\right)^{2} \\
\frac{\mathrm{d} i}{\mathrm{~d} t}=0 \\
\frac{\mathrm{d} \Omega}{\mathrm{d} t}=0 \\
\frac{\mathrm{d} \omega}{\mathrm{d} t}=-\frac{h}{\mu_{\mathrm{com}} e} \cos v p_{0} \frac{1}{B}\left(\frac{r_{0}}{\left|\boldsymbol{r}_{j}\right|}\right)^{2} \\
\frac{\mathrm{d} \bar{M}}{\mathrm{~d} t}=n-\frac{1}{n a}\left(\frac{2 r}{a}-\frac{\left(1-e^{2}\right)}{e} \cos v\right) p_{0} \frac{1}{B}\left(\frac{r_{0}}{\left|\boldsymbol{r}_{j}\right|}\right)^{2}
\end{gathered}
$$

Note that the radial acceleration will always be positive since the outgassing pressure acts only in the outward radial direction. It can be seen that the outgassing pressure affects four of the elements: the semimajor axis $a$, the eccentricity $e$, the argument of perigee $\omega$, and the mean anomaly $\bar{M}$. The $\sin v$ term will be positive while the spacecraft is located along the periapsis to apoapsis portion of its orbit where it has a positive radial component of its velocity. Likewise, the $\sin v$ term will be negative while the spacecraft is located along the apoapsis to periapsis portion of its orbit where it has a negative radial component of its velocity. As seen in semimajor axis and eccentricity equations in Eqs. (40) and (41), the semimajor axis and the eccentricity will increase while the spacecraft's radial velocity is positive and will decrease while the spacecraft's radial velocity is negative as a result of the $\sin v$ term. As noted previously, the parameter $p$ is conserved. This can be proven using the results of a radial acceleration in the Lagrange planetary equations.

$$
\begin{aligned}
\frac{\mathrm{d} p}{\mathrm{~d} t} & =\frac{2 a^{2} e}{h} \sin v p_{0} \frac{1}{B}\left(\frac{r_{0}}{\left|\boldsymbol{r}_{j}\right|}\right)^{2}\left(1-e^{2}\right) \\
& -2 a e \frac{h}{\mu_{\mathrm{com}}} \sin v p_{0} \frac{1}{B}\left(\frac{r_{0}}{\left|\boldsymbol{r}_{j}\right|}\right)^{2}=0
\end{aligned}
$$$$
\frac{\mathrm{d} p}{\mathrm{~d} t}=\frac{\mathrm{d} a}{\mathrm{~d} t}\left(1-e^{2}\right)-2 a e \frac{\mathrm{d} e}{\mathrm{~d} t}
$$

Using these equations, estimates can be computed for changes in orbit due to a jet passage. If it is assumed that the comet spins faster than the orbit travels, the time it takes for the jet to sweep over a spacecraft located at the jet longitude is approximately

$$
\Delta t=\frac{2 \delta}{\omega_{\mathrm{com}} \cos \lambda_{0}}
$$

This leads to impulsive changes in orbital elements of

$$
\begin{array}{r}
\Delta a=\frac{\mathrm{d} a}{\mathrm{~d} t} \Delta t=\frac{2 a^{2} e}{h} \sin \nu p_{0} \frac{1}{B}\left(\frac{r_{0}}{\left|\boldsymbol{r}_{j}\right|}\right)^{2} \frac{2 \delta}{\omega_{\mathrm{com}} \cos \lambda_{0}} \\
\Delta e=\frac{\mathrm{d} e}{\mathrm{~d} t} \Delta t=\frac{h}{\mu_{\mathrm{com}}} \sin \nu p_{0} \frac{1}{B}\left(\frac{r_{0}}{\left|\boldsymbol{r}_{j}\right|}\right)^{2} \frac{2 \delta}{\omega_{\mathrm{com}} \cos \lambda_{0}} \\
\Delta \omega=\frac{\mathrm{d} \omega}{\mathrm{d} t} \Delta t=-\frac{h}{\mu_{\mathrm{com}} e} \cos \nu p_{0} \frac{1}{B}\left(\frac{r_{0}}{\left|\boldsymbol{r}_{j}\right|}\right)^{2} \frac{2 \delta}{\omega_{\mathrm{com}} \cos \lambda_{0}}
\end{array}
$$

Note that if the spacecraft travels opposite to the comet's rotation, the impulse will be less than if the spacecraft travels in the same sense as the comet's rotation because the relative angular rate reversed. For example, a passage of an equatorial jet has an angular rate relative to the jet of $\omega_{\text {com }} \mp n$ depending on direct or retrograde orbit, respectively, such that,

$$
\Delta t=\frac{2 \delta}{\omega_{\mathrm{com}} \mp n}
$$

\section{Simulation}

To test the mathematical model, a simulation was developed. The spacecraft is assumed to follow the trajectory described by solving the standard two-body problem while the spacecraft is not within an outgassing region. The pressure acceleration $\boldsymbol{a}_{p}$ is applied to the spacecraft when it is determined that the angle between the outgassing pressure vector at the same radius as the spacecraft and the spacecraft position vector relative to the jet's virtual center $\theta_{\text {rel }}$ is within a user set angle error tolerance $\Delta \theta_{\text {err }}$ of the half-angle defining the active region's size such that $\theta_{\text {rel }} \leq \delta$ and the boundary is crossed at $\left(\delta-\Delta \theta_{\text {err }}\right) \leq \theta_{\text {rel }} \leq \delta$ illustrated in Fig. 4. The angle $\theta_{\text {rel }}$ is determined by

$$
\theta_{\text {rel }}=\arccos \frac{\boldsymbol{r}_{\mathrm{og}} \cdot \boldsymbol{r}_{j}}{\left\|\boldsymbol{r}_{\mathrm{og}}\right\|\left\|\boldsymbol{r}_{j}\right\|}
$$

where $\boldsymbol{r}$ is the position vector of the spacecraft given in the same coordinate frame as $\boldsymbol{r}_{\mathrm{og}}$.

\section{A. Boundary Detection Precision}

The parameter $\Delta \theta_{\text {err }}$ represents the precision of the instrument used for boundary detection measurements. If Doppler data are used to detect the boundary of the outgassing jet, then the accuracy to which it can unambiguously detect a given change in velocity leads to our modeling of $\Delta \theta_{\text {err }}$ as an estimate of the angle traversed over the time the Doppler data can detect this change. For example, a spacecraft is estimated to traverse approximately $0.1 \mathrm{deg}$ (per minute) while at a radius of $5000 \mathrm{~m}$ and Doppler data can detect changes in velocity of $1 \mathrm{~mm}$ per second over $1 \mathrm{~min}$. Thus, if the outgassing is strong enough to generate this change in speed, the change can be detected in at least a minute of Doppler data. This would isolate the ideal onset of the jet to approximately $0.1 \mathrm{deg}$. This angle increases as the radial distance from the comet increases and decreases as the strength of the jet increases. A diffuse transition region to the full jet strength can also increase the uncertainty of the boundary detect $\Delta \theta_{\text {err }}$.

\section{B. Comet 81/P Wild 2 Model Assumptions}

The outgassing jet model as described is dependent on real values for the outgassing velocity and half-angle as well as the size, shape, and mass of the comet for a simulation. Through the analysis of images taken of comet 81/P Wild 2, locations and orientations of 20 jets have been identified by Sekanina et al. [5] (also located in the

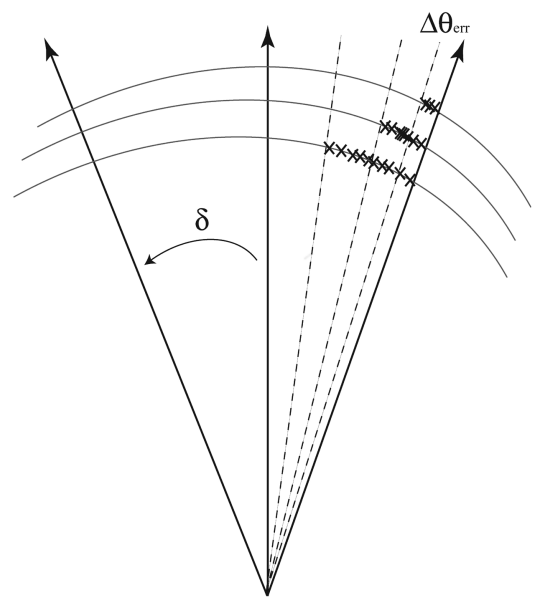

Fig. 4 Illustration of physical meaning of the parameter $\Delta \theta_{\mathrm{err}}$; xs are possible boundary crossing points for different values of $\Delta \theta_{\mathrm{err}}$. 
Table 1 Simulation parameters

\begin{tabular}{llcc}
\hline \hline Variable & \multicolumn{1}{c}{ Description } & Value & Unit \\
\hline$x$ & Comet principle length & 2.75 & $\mathrm{~km}$ \\
$y$ & Comet principle length & 2.00 & $\mathrm{~km}$ \\
$z$ & Comet principle length & 1.65 & $\mathrm{~km}$ \\
$P$ & Comet period & 12 & $\mathrm{~h}$ \\
$V_{\mathrm{og}}$ & Outgassing radial velocity & 0.5 & $\mathrm{~km} / \mathrm{s}$ \\
$\omega_{\text {com }}$ & Comet rotation rate & $\frac{2 \pi}{T}$ & $\mathrm{rad} / \mathrm{s}$ \\
$\delta$ & Jet shape half-angle & 1.5 & $\mathrm{deg}$ \\
$Q_{*}$ & Mass ejection rate & $3.4 \times 10^{6}$ & $\mathrm{~kg} / \mathrm{h}$ \\
$B$ & Spacecraft mass-to-area ratio & $30^{\mathrm{a}}$ & $\mathrm{kg} / \mathrm{m}^{2}$ \\
\hline \hline
\end{tabular}

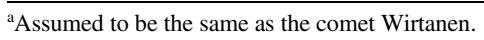

Appendix). The simulated model will use the principle half-lengths defined as 2750, 2000, and $1650 \mathrm{~m}$ [11]. General outgassing velocities for various comets have been estimated to range from $350 \mathrm{~m} / \mathrm{s}$ by Crifo et al. [4] to $900 \mathrm{~m} / \mathrm{s}$ by Combi et al. [8]. In general, the jet half-angle has received less attention but the analysis of the comet Hale-Bopp has indicated discrete jets with estimated halfangles of up to $10 \mathrm{deg}$ [8] and spherical squares with side lengths up to $50 \mathrm{deg}$ have been used by Crifo et al. [4] to describe larger jets. This simulation of the idealized comet $81 \overline{\mathrm{P}} /$ Wild 2 (values located in Table 1) uses the mass ejection rate from Neishtadt et al. [9] with 20 discrete outgassing jets located on the surface in the comet. Each jet is assumed to have a jet half-angle of $1.5 \mathrm{deg}$ and an outgassing velocity of $500 \mathrm{~m} / \mathrm{s}$.

This simulation is seen in Figs. 5- $-\underline{8}$ where the thicker sections of the spacecraft's trajectory are the locations of jet passages. Figure $\underline{8}$ shows the changes in the orbital elements, semimajor axis, and eccentricity, when the spacecraft encounters an outgassing jet. The two jet passages can clearly be identified by the sharp jumps in the orbital elements. Because the spacecraft is located significantly close to the comet, note that the radial impulse assumption may not hold for this simulation.

\section{Estimation of Model}

One of the most important uses of this model is to aid in spacecraft navigation by providing a specific model of outgassing jets with parameters that can be estimated from tracking data. This will both enable future trajectories to be predicted more accurately and provide measurements of scientific interest. Based on Doppler tracking and a standard navigation solution for the spacecraft's trajectory, the jet's basic describing parameters, the jet location and the half-angle $\delta$ and the velocity of the outgassing $V_{\mathrm{og}}$, can be estimated.

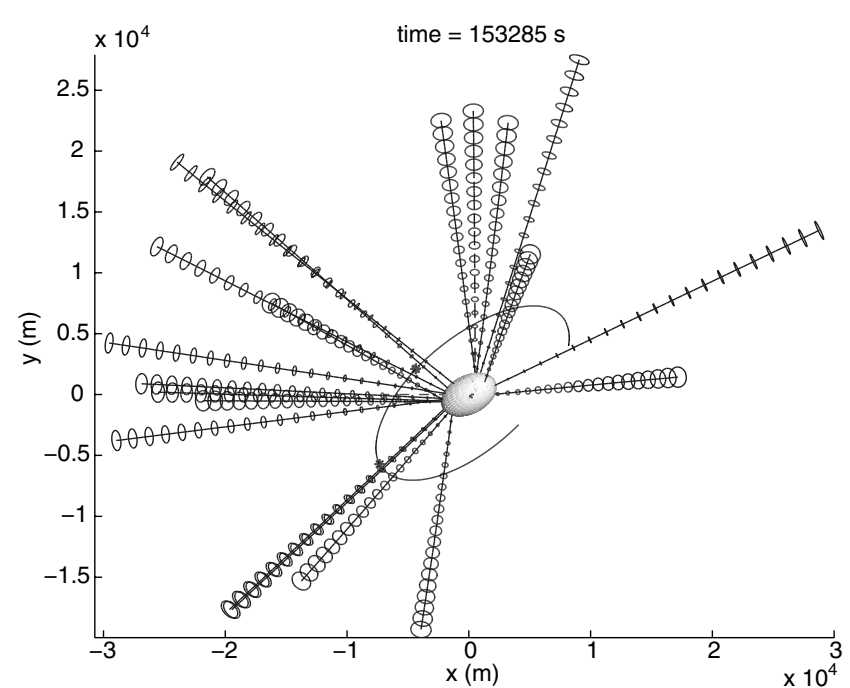

Fig. 5 Spacecraft trajectory in inertial frame for interaction with outgassing jets with a half-angle of $1.5 \mathrm{deg}$ and $V_{\mathrm{og}}$ of $0.5 \mathrm{~km} / \mathrm{s}$ on the idealized comet 81P/Wild 2.

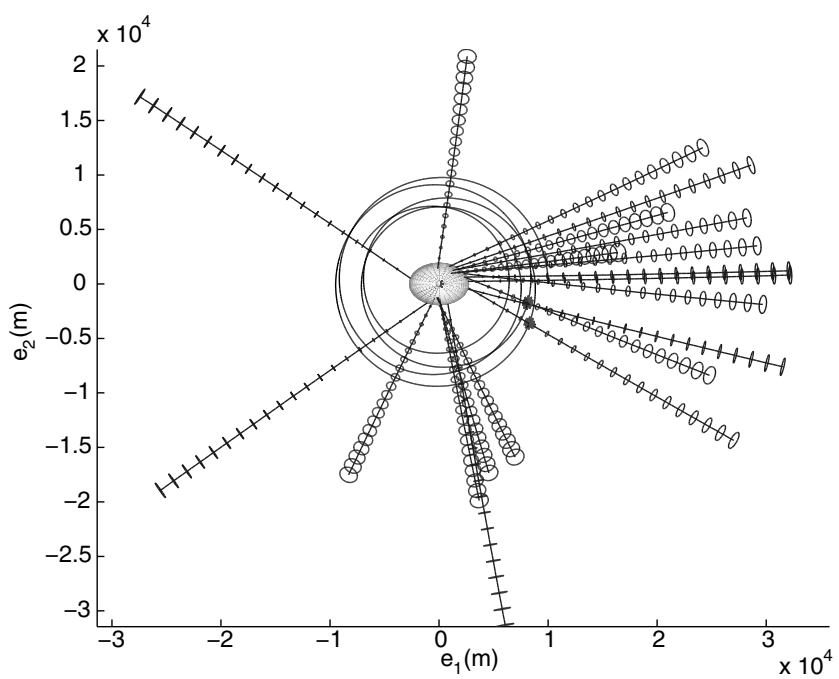

Fig. 6 Spacecraft trajectory in body-fixed frame for interaction with outgassing jets with a half-angle of $1.5 \mathrm{deg}$ and $V_{\mathrm{og}}$ of $0.5 \mathrm{~km} / \mathrm{s}$ on the idealized comet 81P/Wild 2.

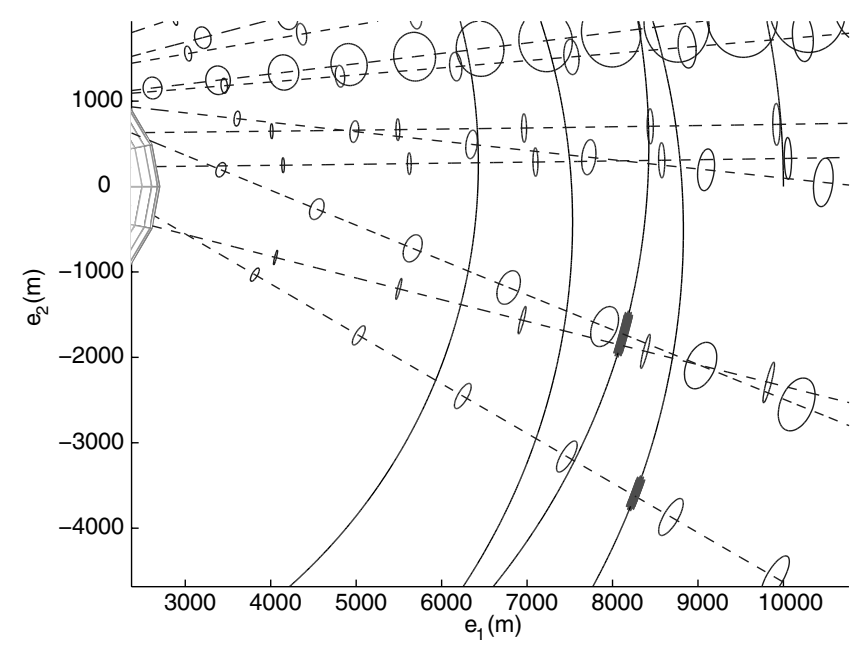

Fig. 7 Spacecraft trajectory in body-fixed frame for interaction with outgassing jets with a half-angle of $1.5 \mathrm{deg}$ and $V_{\mathrm{og}}$ of $0.5 \mathrm{~km} / \mathrm{s}$ on the idealized comet 81P/Wild 2 (detail).
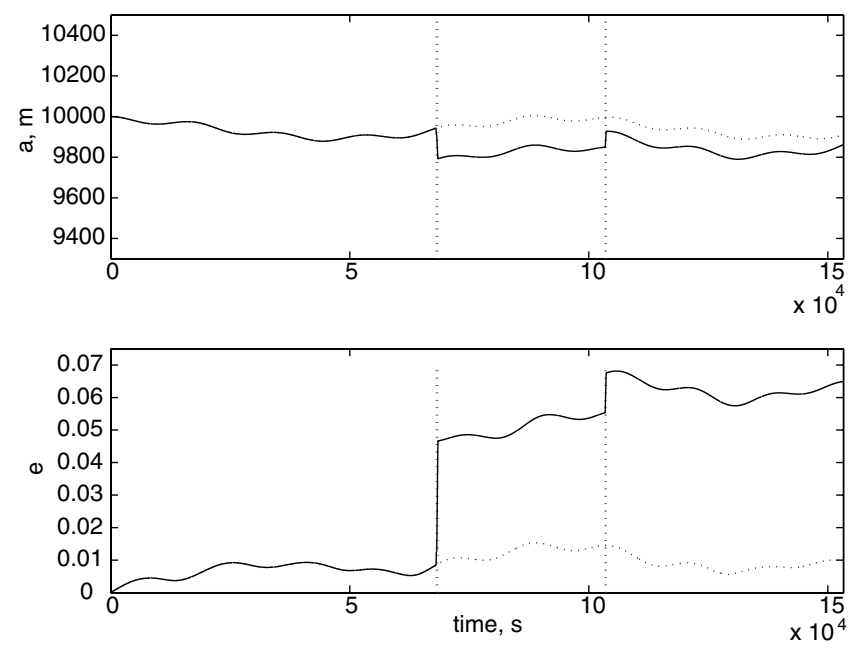

Fig. 8 Semimajor axis and eccentricity for spacecraft with interaction with outgassing jets with a half-angle of $1.5 \mathrm{deg}$ and $V_{\mathrm{og}}$ of $0.5 \mathrm{~km} / \mathrm{s}$ on the idealized comet 81P/Wild 2 with the dotted lines showing the orbital elements for encountering no jets. Variations are because of the nonpoint mass gravity field. 


\section{A. Outgassing Velocity and Acceleration on a Spacecraft}

A spacecraft is assumed to begin passage through an outgassing jet field when the Doppler tracking detects an acceleration larger than $1.667 \times 10^{-5} \mathrm{~m} / \mathrm{s}^{2}$ (the accuracy to which Doppler data can unambiguously detect change in velocity). Because the acceleration is directly measured, if $B$ and the mass flux rate $Q_{j}$ (using a mass spectrometer) can be estimated, then a direct estimate of the outgassing speed can be found using the relationship:

$$
\left|\boldsymbol{a}_{p}\right|=Q_{j} V_{\mathrm{og}} \frac{1}{B}\left(\frac{r_{0}}{\left|\boldsymbol{r}_{j}\right|}\right)^{2}
$$

In addition to estimating the outgassing velocity, this detection can also be used to identify boundary crossings of the jet outgassing field used in the estimation of other jet parameters assuming that a navigation solution for the spacecraft is available.

The heliocentric radius of the comet is also important at the measurement times. By assuming an outgassing velocity of $0.5 \mathrm{~km} / \mathrm{s}$ in the model, the magnitude of the outgassing acceleration can be detected by Doppler tracking when the model comet's orbital radius is less then $3 \mathrm{AU}$ as seen in Fig. 9. The spikes in the spacecraft's acceleration components (seen in Fig. 10 from simulation data of a comet at approximately $1.5 \mathrm{AU}$ ) illustrate jet passages. Because the magnitude of the acceleration spikes are on the order of $10^{-5} \mathrm{~m} / \mathrm{s}^{2}$, the jet passages are identifiable by the Doppler tracking. Note that these acceleration magnitudes are theoretical and based on the outgassing jet models. To date there have been no mission data available to verify such behavior.

\section{B. Jet Location and Half-Angle $\delta$}

With multiple jets emanating from the surface of the comet, it is necessary to identify each crossing with the appropriate jet location. Note that there is an underlying assumption that no outgassing jets intersect for the estimation methods discussed. The jet boundary crossings (discussed previously) naturally occur in pairs, one crossing classified as entering and one as exiting the outgassing field. These pairs can be mapped to the surface of the comet in the body-fixed frame as unit vectors $\hat{u}_{i}$ that lie on the boundary of the jet if the rotation of the comet and the outgassing velocity $V_{\text {og }}$ is assumed to be known. The time since ejection $s$ can be found using the assumed outgassing velocity and the radial distance from the surface of the comet which yields the angular rotation of the comet from the rotation rate, $\omega_{\text {com. }}$. Rotating the comet back in time and moving the crossing point to the surface of the comet gives the mapping method to a body-fixed frame.

After each pair has been mapped to the comet surface, they are identified with a possible jet location by checking the proximity of the unit vector which defines the pair's bisector with possible jets' estimated centerlines. If the bisecting unit vector falls within the jet's

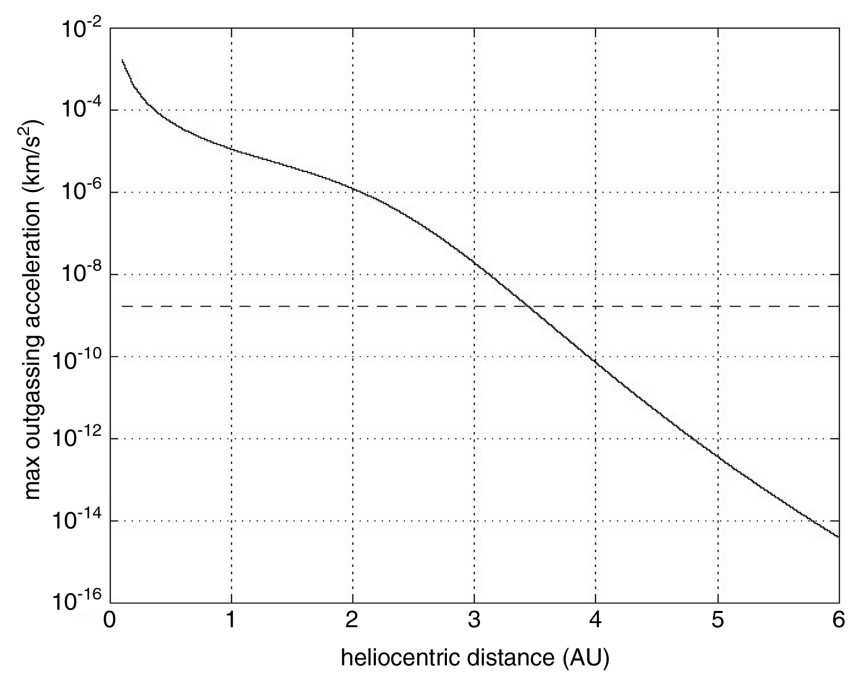

Fig. 9 Outgassing acceleration as a function of comet orbital radius (Doppler tracking threshold shown as a dotted line).

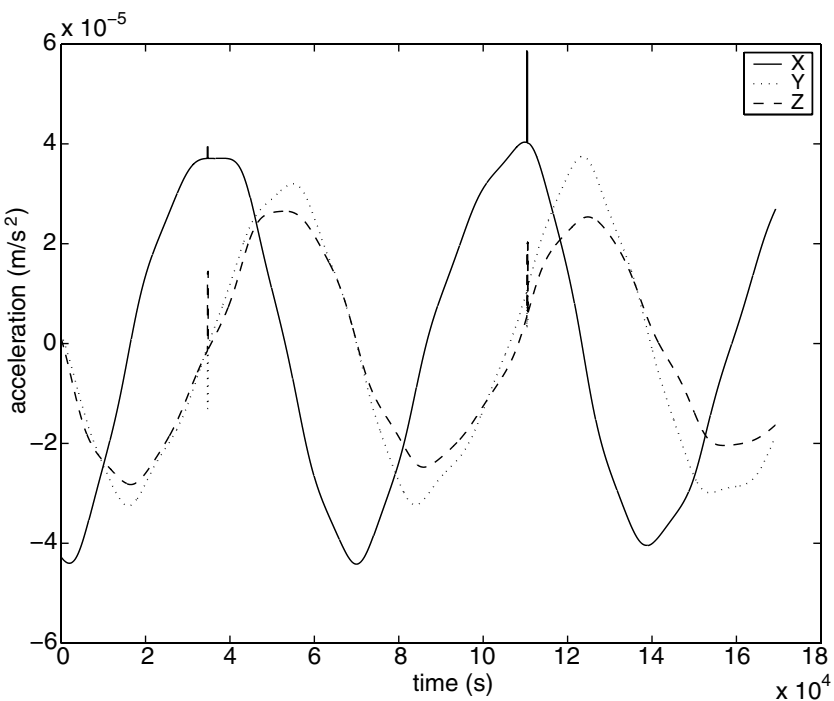

Fig. 10 Spacecraft acceleration components in an inertial frame.

estimated size (defined as the estimated half-angle with some allowable tolerance), then the pair is grouped with other identified pairs for that possible jet location. If the bisector unit vector does not match with an existing possible jet location, the pair's bisecting unit vector defines a new possible jet location to be tested for subsequent crossings.

Once a crossing pair has been identified with a possible jet location, the number of pairs identified with that jet location determines the estimation process. If only a single pair has been identified, then the jet centerline location is assumed to be the bisecting unit vector, and the half-angle of the jet is estimated as half of the angle between the pair. If multiple pairs are associated with a possible jet location, there exist many possible methods to estimate the jet location and half-angle. Here three methods are explored, where two methods are based on the geometry of the circular cross section and the third uses the directly determined unit vectors. Each method allows for the spacecraft to fly through at different radii and allows for curved paths through the outgassing field since the crossings are mapped to body-fixed unit vectors. The first geometrical approach needs two pairs of crossings to estimate the position and jet half-angle where the pairs are connected to form two chords. The crossing of the bisecting perpendiculars to these chords will give an estimate of the center of the jet. Using Pythagoras' theorem on one of the chords yields the estimate of the half-angle as seen in Fig. 11. If the spacecraft is known to have crossed the centerline of the outgassing jet, then the chord constructed is the diameter of the jet cross section and reduces to the method used for a single pair crossing.

The second geometrical approach requires only one and one-half crossing pairs of the jet outgassing field. Using the three unit vectors in the body-fixed frame as vertices of a triangle, the geometrical formula for finding the radius of a circumscribed circle about a triangle can be used to estimate the jet half-angle $\delta$ as seen in Fig. 12.

$$
\begin{gathered}
\sin \delta=\frac{1}{2} \frac{a_{t} b_{t} c_{t}}{\sqrt{s\left(s-a_{t}\right)\left(s-b_{t}\right)\left(s-c_{t}\right)}} \\
s=\frac{1}{2}\left(a_{t}+b_{t}+c_{t}\right)
\end{gathered}
$$

where $a_{t}, b_{t}$, and $c_{t}$ are the chord lengths created by connecting the three crossings. Once the half-angle is known, the center of the jet can be inferred by finding the common intersection point of circle of radius $\sin \delta$ drawn about each crossing point. This approach could be used if one of the boundary crossings is ill defined.

The third approach based on vector algebra is the most general and allows for noncircular cross sections of the outgassing jet to be ideally estimated. Using the mapped pairs of unit vectors $\hat{u}_{i}$ in the body-fixed frame (illustrated in Fig. 13), a unit vector pointing to the 


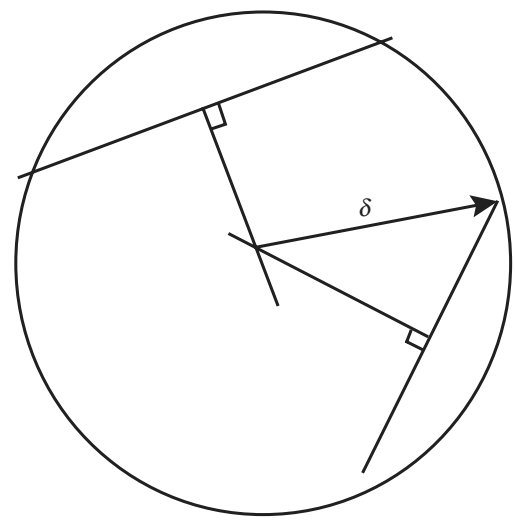

Fig. 11 Perpendicular bisectors method of estimating jet half-angle.

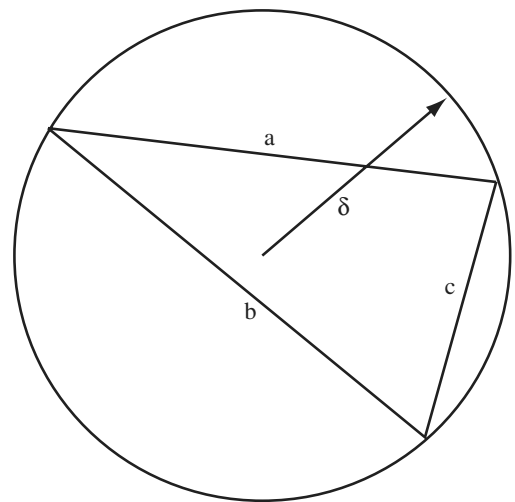

Fig. 12 Circumscribed triangle method of estimating jet half-angle.

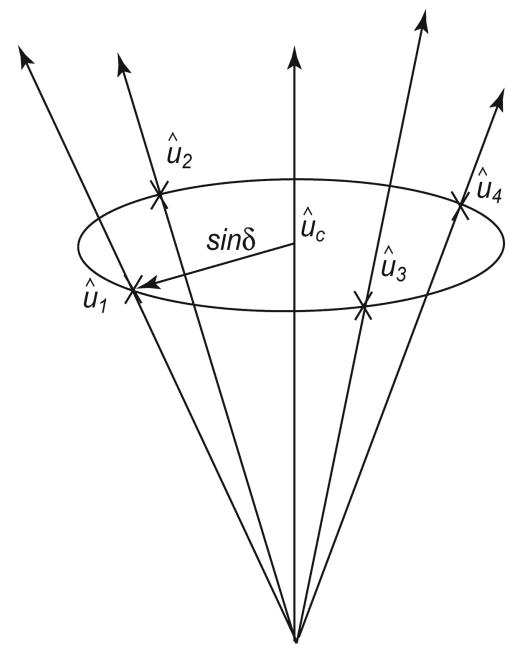

Fig. 13 Body-fixed unit vectors method of estimating jet half-angles.

jet location in the direction of the estimated center $\hat{u}_{c}$ of the cross section can be defined. The nominal cone angle is found as

$$
\hat{u}_{i} \cdot \hat{u}_{c}=\cos (\delta)
$$

A pair of boundary crossings will produce a plane of possible solutions to $\hat{u}_{c}$. The position of the center of the outgassing jet can be estimated by a third crossing which gives a unique solution to $\hat{u}_{c}$ and thus the half-angle $\delta$.

This estimate for $\hat{u}_{c}$, and hence $\delta$, can be refined when subsequent boundary crossings occur by using a least-squares approach, where the cost function $J$ is defined as

$$
J=\frac{1}{2} \sum_{i=1}^{N}\left(\hat{u}_{i} \cdot \hat{u}_{c}-\cos \delta\right)^{2}
$$

Taking the partial derivative with respect to the center unit vector and setting it equal to zero yields an estimate for $\hat{u}_{c}$.

$$
\begin{gathered}
\frac{\partial J}{\partial \hat{u}_{c}}=\sum_{i=1}^{N}\left(\hat{u}_{i} \cdot \hat{u}_{c}-\cos \delta\right) \hat{u}_{i}=0 \\
\boldsymbol{u}_{c}=\cos \delta\left(\sum_{i=1}^{N} \hat{u}_{i} \hat{u}_{i}\right)^{-1} \sum \hat{u}_{i}
\end{gathered}
$$

This solution should point in the direction of the proper solution, but may not be a unit vector. Normalizing the estimate for $\boldsymbol{u}_{c}$ yields a function only of the boundary crossing unit vectors.

$$
\hat{u}_{c}=\frac{\left(\sum_{i=1}^{N} \hat{u}_{i} \hat{u}_{i}\right)^{-1} \sum \hat{u}_{i}}{\left|\left(\sum_{i=1}^{N} \hat{u}_{i} \hat{u}_{i}\right)^{-1} \sum \hat{u}_{i}\right|}
$$

Note that for $N<3$, the term $\sum_{i=1}^{N} \hat{u}_{i} \hat{u}_{i}$ will not be invertible in general, but that for $N \geq 3$ for a properly chosen set of $\hat{u}_{i}$ will produce a unique solution. Next, taking the partial derivative of the cost function with respect to the jet half-angle $\delta$ yields an estimate for the half-angle which is dependent on the estimate for the center unit vector found in Eq. (61).

$$
\begin{gathered}
\frac{\partial J}{\partial \delta}=\sum_{i=1}^{N}\left(\hat{u}_{i} \cdot \hat{u}_{c}-\cos \delta\right) \sin \delta=0 \\
\cos \delta=\frac{1}{N}\left(\sum_{i=1}^{N} \hat{u}_{i}\right) \cdot \hat{u}_{c} \\
\delta=\arccos \left(\frac{1}{N} \hat{u}_{c} \cdot \sum_{i=1}^{N} \hat{u}_{i}\right)
\end{gathered}
$$

These new estimates for the jet centerline unit vector and half-angle are used to identify subsequent jet crossing pairs with the jet. This iterative process allows for the mapped pairs to be identified properly to the correct jet while constantly updating the estimated location and half-angle of each possible jet.

Once the location and half-angle of the jets have been estimated, the mapped boundary crossing vectors can be intersected with the surface of the comet to define the boundary of an active surface patch associated with the jet. Correlating this surface patch with images of the comet's surface can yield insight into the orientation or source of the jet from surface features such as cavities or sharp inclines. The errors in these estimations can be caused by uncertainty in the spacecraft's position, uncertainty in the comet's rotation, from lack of detailed knowledge of the outgassing jet's structure, or from the assumed outgassing velocity. Uncertainty in the spacecraft's position and in the comet's rotation can produce errors in the mapped unit vectors. Lack of knowledge of the jet's structure can lead to errors in the identification of jet locations. One such scenario is the jet's outgassing pressure profile not being a solid cone of pressure but is instead a hollow cone producing more boundary crossings than actually exist, yielding the appearance of crossing two jets instead of one. An inaccurate velocity for the outgassing will produce errors in the jet location on the surface of the comet. These types of errors need to be explored further.

It is noted that these methods are best applied while the spacecraft is close to the comet body where the geometry of the outgassing jet is well defined. As the spacecraft travels farther from the comet, the detection of the outgassing jet boundaries will become increasingly difficult due to the jet pressure weakening as a function of the distance from the comet body.

\section{Estimation Simulation}

Using the model simulation, the boundary crossing positions and times are collected to estimate the jet parameters. For the estimation of the jet half-angle $\delta$, the estimation simulation uses the unit vector least-squares approach to estimate the jet center unit vector and the jet 
half-angle assuming that the outgassing velocity and comet rotation is already known. Note that the locations of possible jets are not assumed and are also estimated by the multiple jet identification method discussed in the previous section. The simulation uses halfangles of $1.5 \mathrm{deg}$ and outgassing velocities of $0.5 \mathrm{~km} / \mathrm{s}$ for the jets (seen in Fig. 14). The jet half-angle is estimated for different angle error tolerances $\Delta \theta_{\text {err }}$, replicating how well the boundary can be detected. The first trajectory point which was determined to fall within the $\theta_{\text {rel }}$ conditions $\left(\delta-\Delta \theta_{\text {err }}\right) \leq \theta_{\text {rel }} \leq \delta$ was used as the boundary crossing point. Various angle error tolerance values ranging from 0.1 to $1 \mathrm{deg}$ were used for comparison and also to account for jet boundaries which may be ill defined and therefore take a longer integration time to detect. The outgassing velocity used is assumed to be known from previous estimations. For the half-angle estimate, a small angle error, defined by the size of $\Delta \theta_{\text {err }}$, in the measurement results, in general, in a smaller half-angle estimate error as seen in Table 2. The jet half-angles which remained constant with varying angle error tolerance are caused by the boundary crossings lying within the smallest angle error tolerance for all cases. The exceptions are caused by the time step used in the simulated data. The errors in the estimation can be caused by uncertainty in the spacecraft's position, uncertainty in the comet's rotation, or from lack of detailed knowledge of the outgassing jet's structure discussed previously.

\section{Estimation Applications}

The described estimation methods have many applications as navigation tools for a spacecraft. The estimates can be used to identify jets and map their locations on the surface of the comet as well as determine their size. These parameters can provide better trajectory fits and predictions of a spacecraft in orbit about a comet. The location of the jet also allows for targeted scientific measurements, turning on measurement instruments only when the spacecraft is in or near a jet outgassing field or avoidance of jets for imaging or landing missions. In addition to scientific measurements, jets may be targeted as a passive control of the spacecraft. A passage of a jet can be used to change the semimajor axis and eccentricity of the spacecraft's orbit without the orbital maneuver planning and component safety checks associated with using thrusters.

In conjunction with mass spectrometer measurements, the gas velocity can be determined with simple relationships. This estimation also holds for a given gas velocity from which the gas mass flux can be determined. Applications of the outgassing field and jet structure can also be obtained once the field boundary has been crossed. The spacecraft can measure the variation of the outgassing within a jet's field determining if it is a solid cone structure as assumed in the model or actually a cone with an inactive or less active interior. Multiple passes can uncover complex jet boundaries that may be related to the outgassing conditions at the comet surface.

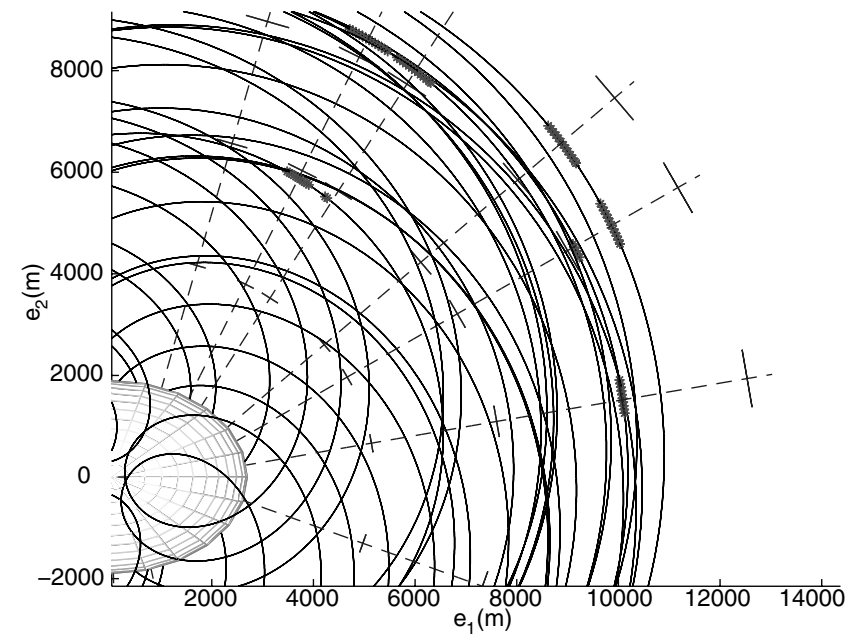

Fig. 14 Simulation of jets with half-angle of $1.5 \mathrm{deg}$ and outgassing velocity of $0.5 \mathrm{~km} / \mathrm{s}$ (detail).
Table 2 Outgassing half-angle estimation (deg)

\begin{tabular}{lcccc}
\hline \hline & & \multicolumn{3}{c}{ Angle error tolerance } \\
\cline { 3 - 5 } Jet no. & Crossings pts. & 1 deg & $0.5 \mathrm{deg}$ & $0.1 \mathrm{deg}$ \\
\hline 1 & 2 & 2.3337 & 2.3337 & 2.5458 \\
2 & 4 & 2.3120 & 2.4327 & 2.8134 \\
3 & 4 & $0.6354 \mathrm{a}$ & 2.2614 & 2.4815 \\
4 & 2 & 1.9073 & 1.9073 & 1.9073 \\
5 & 2 & 0.4227 & 0.4227 & 0.4227 \\
6 & 2 & 1.6908 & 1.6908 & 1.6908 \\
7 & 2 & 1.9236 & 1.9236 & 2.1373 \\
8 & 4 & 2.9108 & 2.9108 & 1.9160 \\
9 & 2 & 2.4646 & 2.4646 & 2.2406 \\
10 & 2 & 0.4206 & 0.6309 & 0.8412 \\
11 & 2 & 2.5279 & 2.5279 & 2.5279 \\
12 & 2 & 1.6857 & 1.4750 & 1.6857 \\
\hline \hline
\end{tabular}

${ }^{a}$ Estimated with two crossing points.

\section{Conclusions}

This paper has presented a simple mathematical model for a single with constant half-angle $\delta$, using a uniform ellipsoidal model of the comet. The model of the comet was simulated using 20 discrete jets to verify the results found in the changes in the orbital elements. From the small changes in the orbital parameter and the orbital energy, it was shown that if the spacecraft has a negative radial velocity component, the outgassing jet will decrease the semimajor axis and eccentricity, and if the spacecraft has a positive radial velocity component, the outgassing jet will tend to make the orbit more eccentric and increase the semimajor axis for a radial impulse when the spacecraft is located significantly far from the comet.

Estimation methods were presented for the outgassing velocity, the half-angle, and the locations of jets. The outgassing velocity can be determined by taking a measurement of the mass flux and the change in the spacecraft's acceleration. The half-angle can be estimated by using boundary crossing points mapped to the surface of the comet. The method for estimating the half-angle of the jet includes an algorithm for identifying jet locations when multiple jet outgassing fields have been crossed. For the half-angle estimation method simulated, a small angle error in the measurement results in general in a small half-angle estimate error.

\section{Appendix}

The jet locations and orientations in Table $\underline{\mathrm{A} 1}$ are from Sekanina et al. [ㅁ]

Table A1 Jet locations and orientations

\begin{tabular}{lrrrr}
\hline \hline \multicolumn{3}{c}{ Orientation angles, deg } & \multicolumn{2}{c}{ Jet location angles, deg } \\
Jet & \multicolumn{1}{c}{$\psi_{\text {or }}$} & $\lambda_{\text {or }}$ & $\phi_{\text {loc }}$ & $\lambda_{\text {loc }}$ \\
\hline$\alpha$ & 161 & 4 & 169 & 1 \\
$\beta$ & -7 & 36 & 16 & 14 \\
$\gamma$ & 25 & 26 & 38 & 13 \\
$\delta$ & 22 & 59 & 42 & 38 \\
$\epsilon$ & -15 & -20 & 353 & -7 \\
$\zeta$ & 9 & 23 & 23 & 9 \\
$\eta$ & -65 & 3 & 294 & 2 \\
$\theta$ & -130 & 4 & 238 & 2 \\
$\kappa$ & 40 & 31 & 60 & 19 \\
$\lambda$ & 30 & 47 & 45 & 28 \\
$\mu$ & -59 & 56 & 266 & 43 \\
$\nu$ & -49 & 57 & 260 & 43 \\
$\xi$ & 20 & 25 & 27 & 10 \\
$\pi$ & 34 & 17 & 45 & 9 \\
$\rho$ & -64 & 51 & 272 & 38 \\
$\sigma$ & 97 & 49 & 95 & 36 \\
$\tau$ & -101 & 53 & 252 & 39 \\
$\phi$ & 16 & -10 & 14 & -3 \\
$\chi$ & 16 & -10 & 5 & -3 \\
$\psi$ & 1 & -7 & 350 & -2 \\
\hline \hline
\end{tabular}




\section{Acknowledgment}

This research was funded, in part, by a grant from the Jet Propulsion Laboratory/California Institute of Technology Director's Research and Development Fund.

\section{References}

[1] Miller, J. K., Weeks, C. J., and Wood, L. J., "Orbit Determination Strategy and Accuracy for a Comet Rendezvous Mission," Journal of Guidance, Control, and Dynamics, Vol. 13, No. 5, 1990, pp. 775-784.

[2] Weeks, C. J., "The Effect of Comet Outgassing and Dust Emission on the Navigation of an Orbiting Spacecraft," Journal of Astronautical Sciences, Vol. 43, No. 3, 1995, pp. 327-343.

[3] Scheeres, D. J., Marzari, F., Tomasella, L., and Vanzani, V., "ROSETTA Mission: Satellite Orbits Around a Cometary Nucleus," Planetary and Space Science, Vol. 46, Nos. 6/7, 1998, pp. 649-671.

[4] Crifo, J. F., Itkin, A. L., and Rodionov, A. V., "The Near-Nucleus Coma Formed by Interacting Dusty Gas Jets Effusing from a Cometary Nucleus: I," Icarus, Vol. 116, No. 1, 1995, pp. 77-112.

[5] Sekanina, Z., Brownlee, D. E., Economou, T. E., Tuzzolina, A. J., and
Green, S. F., "Modeling the Nucleus and Jets of Comet 81P/Wild 2 Based on the Stardust Encounter Data," Science, Vol. 304, No. 5678, 2004, pp. 1769-1774.

[6] 2006 Solar System Exploration Roadmap, NASA, March 2006, http://solarsystem.nasa.gov/multimedia/downloads.cfm [retrieved Aug. 2006].

[7] Scheeres, D. J., "Dynamics About Uniformly Rotating Tri-Axial Ellipsoids. Applications to Asteroids," Icarus, Vol. 110, 1994, pp. 225 238.

[8] Combi, M. R., Kabin, K., DeZeeuw, D. L., and Gombosi, T. I., "DustGas Interrelations in Comets: Observations and Theory," Earth, Moon, and Planets, Vol. 79, 1997, pp. 275-306.

[9] Neishtadt, A. I., Scheeres, D. J., Sidorenko, V. V., and Vasiliev, A. A., "Evolution of Comet Nucleus Rotation," Icarus, Vol. 157, 2002, pp. 205-218.

[10] Marsden, B. G., Sekanina, Z., and Yeomans, D. K., "Comets and Nongravitational Forces," Astronomical Journal, Vol. 78, No. 173, 1973, pp. 211-225.

[11] Stardust SPICE Data [online database], Jet Propulsion Laboratory, 2005 , ftp://naif.jpl.nasa.gov/pub/naif/SDU/kernels/pck/ sdu_wild2_v01.tpc [retrieved May 2006]. 